\title{
Building a vector model
}

representation of a two-axis laser

scanhead using numerical analysis

for simulation purposes

\author{
Journal Article \\ Author(s): \\ Eberle, Gregory; Dold, Claus; Wegener, Konrad \\ Publication date: \\ 2013 \\ Permanent link: \\ https://doi.org/10.3929/ethz-a-010268332
}

Rights / license:

In Copyright - Non-Commercial Use Permitted

Originally published in:

International Journal of Modelling, Identification and Control 20(3), https://doi.org/10.1504/IJMIC.2013.057131 


\title{
Building a vector model representation of a two- axis laser scanhead using numerical analysis for simulation purposes
}

\author{
Gregory Eberle* \\ Institute of Machine Tools and Manufacturing \\ Swiss Federal Institute of Technology Zurich \\ Tannenstrasse 3, Zurich, 8092, Switzerland \\ E-mail: eberle@iwf.mavt.ethz.ch \\ *Corresponding author
}

\section{Claus Dold}

inspire AG

Swiss Federal Institute of Technology Zurich

Tannenstrasse 3, Zurich, 8092, Switzerland

E-mail: claus.dold@inspire.ethz.ch

\section{Konrad Wegener}

Institute of Machine Tools and Manufacturing

Swiss Federal Institute of Technology Zurich

Tannenstrasse 3, Zurich, 8092, Switzerland

E-mail: wegener@iwf.mavt.ethz.ch

\begin{abstract}
A vector model representing laser beam propagation into a two-axis laser scanhead is proposed. The model based on mathematical principles is classified as a stochastic model in which at least one of the input or output variables is probabilistic. The purpose of the model is to understand the behaviour of the scanhead system particularly in relation to the errors incurred from its design and the laser beam. Using this model, simulation looks into reproducing the marking results of a commercially available scanhead, effects of aperture misalignment, 3D ray tracing and Gaussian beam relations.
\end{abstract}

Keywords: Matlab; two-axis scanning; galvanometer; laser scanning; scanhead; ray tracing; vector model; Gaussian beam; optical distortions; misalignment; laser propagation.

Reference to this paper should be made as follows: Eberle, G., Dold, C., and Wegener, K. (2013) 'Building a vector model representation of a two-axis laser scanhead using numerical analysis for simulation purposes', Int. J. Modelling, Identification and Control, Vol. x, No. x, pp.xxx-xxx.

Biographical notes: Gregory Eberle completed in 2009 his Bachelor of Engineering at the University of Ontario Institute of Technology, Canada. Subsequently, he completed in 2011 his Master of Science at the Swiss Federal Institute of Technology Zurich, Switzerland. His competencies are in the field of laser scanning technologies and techniques, and laser material microprocessing. He is now in the process of continuing his doctorate studies with Swiss Federal Institute of Technology Zurich in the field of laser processing of ultra-hard materials.

Claus Dold has a dual Bachelors degree in architecture and mechanical engineering from the Swiss Federal Institute of Technology Zurich. He completed his Masters degree and is finishing his final year of his doctorate studies at the Swiss Federal Institute of Technology Zurich. His competencies include laser material processing using ultrafast laser systems, machining and surface modifications of hard materials typically in the area of tooling surfaces and coatings, and analysis and simulation of laser beam material interaction.

Konrad Wegener completed his doctorate at the Technical University of Braunschweig, Germany. Subsequently, he was involved with the optimization, processing planning, standardization and introduction of new technologies at Schuler Presses GmbH \& Co. KG. He was involved in the 
growth, development and production of large welding machines for heavy industries. He is now the head of the Institute of Machine Tools and Manufacturing and professor at the Swiss Federal Institute of Technology Zurich since 2003. His competencies are in the field of machine tools, manufacturing processes and process chains, and the development, evaluation and optimization of production machinery.

\section{Introduction}

A focused laser beam can only be useful to the user if it can be effectively, safely and accurately controlled to follow a defined motion. A common solution is the use of an optical scanning technique designated to a specific application. The most common and efficient scanning technique in laser material processing is galvanometer scanning. Galvanometer scanners and their auxiliary components are assembled in what is referred to as a scanhead. Hence, a laser scanhead is a device which focuses a laser beam and guides the focused spot according to the user's targeted outcome by means of an objective lens and mirrors respectively.

a)

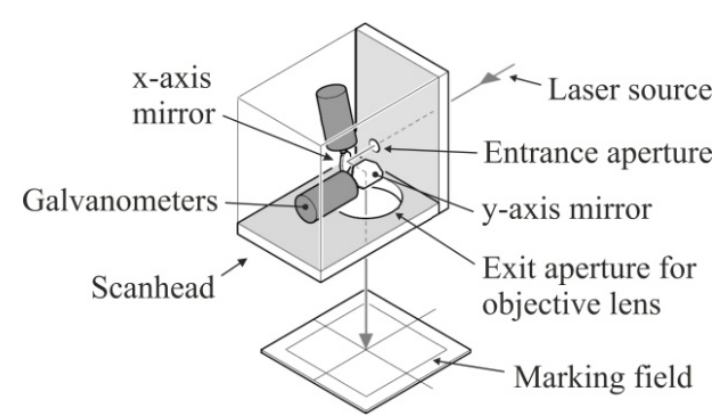

b)

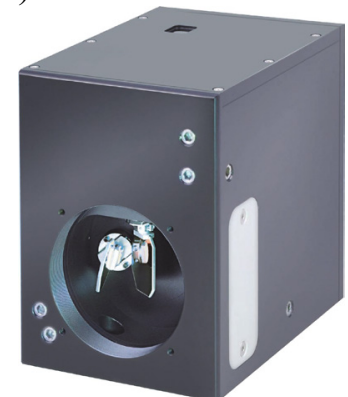

Figure 1 Principle arrangement for 2D galvanometer scanning (a) and an industrial realization of a scanhead, e.g. Raylase SuperScan-LD (b) (Raylase, 2012)

There has been a wide range of studies in terms of scanhead functionality, particularly in marking field correction algorithms. Thus, there are many resources and different approaches as how to simulate a two-axis scanhead to determine the output image on the marking field and subsequently outlining a method of correction. In the ideal approach, it is necessary to model the physical layout of a scanhead in order to determine the output image and thus provide a means of field correction. The following explains current results with respect to modelling and/or simulating laser scanning methods:

Verboven (1988) uses trigonometric analysis to determine the image on the marking field, and introduces a method to electronically correct the position of the imaged distortion by establishing at which angles the mirrors must be rotated to attain the desired image values using a fifth-order series expansion. Li and Katz $(1995,2008)$ present an approach of modelling the generated marking field results of various types of scanning architectures (e.g. galvanometer, polygon, single mirror, etc.) analytically. Vector analysis is carried out in order to understand and predict the image, optical distortions, spot characteristics, and linearity results on the marking field. The developed algorithms and coordinate system are unified and can be applied to other architectures of scanning systems independent of the number of mirrors and arrangement thereof. Hafez, Sidler and Salathé (2003) use vector analysis to firstly determine beam propagation, outline the dominating parameters that cause image distortion and finally compensation algorithms for these distortions. The model is based upon one scanning mirror which has two degrees of freedom; the mirror is able to tip and tilt the laser beam onto the marking field. Bessmeltsev, Goloshevsky and Smirnov (2010) offer an advanced explanation of integrating the developed model into the controller of each respective galvanometer of a scan system by creating a relation between the position of the laser beam on the marking field, the angular deflections of the mirrors and the applied voltages to the galvanometers. The model is unique since it 
takes into account a marking field that is in motion, e.g. a conveyor line. Tamura et al. (1994) develop a robust model for the application of high precision 3D measurements. The developed model encompasses principles such as coordinate transformations of the laser beam and marking field, rotation about an arbitrary axis and calibration. Gandhi and Deshmukh (2010) carry out an optical analysis of four types of scanning architectures. Matlab ${ }^{\circledR}$ and OSLO ${ }^{\circledR}$ simulation programs are used to compare and develop a new optical scanning scheme for the application of microstereolithography. Ray analysis, spot size and intensity profile determination, resolution of spot positioning through induced misalignments, scan speed and linear range of scanning are predicted. Bai and Wang (2011) model the dynamics of a gimbal scanner for a laser tracking system using Lagrange-Euler equations of motion. The three coordinate frames and axes displacement are considered, similar to the displacement found in galvanometer scanning. Additionally, simplifications to characterise the unique kinematic architecture of a gimbal is proposed. The model is used to develop a control system for each scanning motor. However, an in-depth explicit explanation of mathematically modelling and simulating a twoaxis scanhead using Matlab ${ }^{\circledR}$ has yet to be presented.

\section{$2 \quad$ Model setup}

The main goal of this paper is to develop a mathematical model which represents a two-axis galvanometer scanning system. Additionally, the model is expected to simulate the image on the marking field, observe the effects of beam aperture misalignment, and observe the change in beam shape and intensity during mirror rotation. This allows for an improved understanding into the behaviour of the system particularly in relation to the errors from its design and the laser beam. The model of this discussion will focus mainly on a mathematical approach. Therefore, it is classified as a stochastic model in which at least one of the input or output variables is probabilistic (Garrido, 2009). Since the behaviour of scanhead mirrors are known from experimentation, the behaviour of the scanhead as a whole is investigated in order to vary and observe the inputs and outputs respectively, as well as offer insight to predictions.

The derived model is based upon many of the fundamental principles as discussed in the previous section. The use of vector analysis and principles according to Hafez et al. and Tamura et al. respectively will form the basis of modelling a scanhead based on a pre-objective scanning architecture. This allows for the analysis of a laser beam striking the scanning mirrors in an arbitrary position, takes into account mirror thickness distortion, does not assume geometric symmetries and offers clear insight into the laser beams projected path at each stage of its propagation. There are a number of assumptions that are made, but simplifications in terms of the geometric design of the system are not present. The model is to replicate the functionality of the Raylase Superscan Low Drift (LD) scanhead. The resting angle of incidence of the $\mathrm{x}$-mirror and y-mirror is $45^{\circ}$ and $38^{\circ}$ respectively. Furthermore, the $\mathrm{x}$-mirror is rotated about the $\mathrm{x}$-axis $11.5^{\circ}$ clockwise. This allows for a more compact design, i.e. the beam displacement is reduced. Finally, the objective lens follows the ftheta condition. The procedure of how to model beam propagation into and out of a scanhead will now be explained in terms of vector analysis such that one refers to Figure 2 for variable notations. 


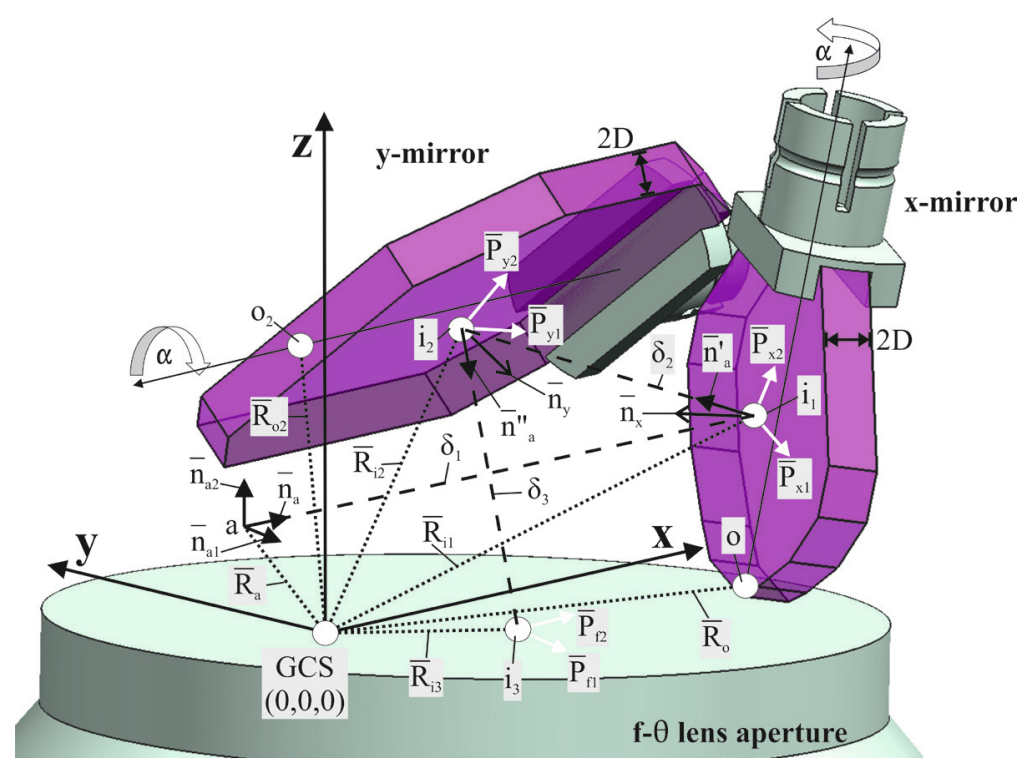

Figure 2 Vector and coordinate system notations

The vector from the origin of the global coordinate system (GCS) to the first point of impact, $i_{1}$, on the $\mathrm{x}$-mirror is given by:

$$
\vec{R}_{\tilde{i}_{1}}=\vec{R}_{a}+\delta_{1} \cdot \vec{n}_{a}
$$

such that $\vec{n}_{a}$ is the unit vector in the direction of propagation of the laser beam, $(1,0,0), \delta_{1}$ is the magnitude of laser beam propagation distance between its initial start position, e.g. $(5,5,5)$, and point of impact, $i_{1}$, and $\vec{R}_{a}$ is the vector from the origin of the GCS to the start position, point $a$. The scalar, $\delta_{1}$, is given by:

$$
\delta_{1}=\frac{\vec{n}_{x} \cdot\left(\vec{R}_{o}-\vec{R}_{a}\right)+D}{\vec{n}_{x} \cdot \vec{n}_{a}}
$$

such that $D$ is the half-width of the mirror's thickness, $\vec{n}_{x}$ is the normal vector of the x-mirror's surface and $\vec{R}_{o}$ is the vector from the origin of the GCS to a point along rotation axis of the x-mirror. In order to calculate $\vec{n}_{x}$, three arbitrary points are selected from the surface of the x-mirror. In turn, two vectors are generated. By taking the cross product and then normalizing, one is able to obtain the normal unit vector of the $\mathrm{x}$-mirror. Therefore:

$$
\vec{n}_{x}=\left\|\vec{P}_{x_{1}} \times \vec{P}_{x_{2}}\right\|
$$

In doing so, it is assumed that the surface of each mirror is an infinite plane. Using Equation 2 and Equation 3, one can calculate the point of impact, $i_{1}$, of the laser beam on the x-mirror with respect to the GCS using Equation 1. Upon calculating $\vec{R}_{i_{1}}$, one can proceed to calculating the vector from the origin of the GCS to the point of impact, $i_{2}$, on the y-mirror which is given by:

$$
\vec{R}_{i_{2}}=\vec{R}_{i_{1}}+\delta_{2} \cdot \vec{n}_{a}^{r}
$$

such that $\vec{n}_{\alpha}^{*}$ is the unit vector along the reflected beam, i.e. coincident to $\delta_{2}$. It is noticeable that the unit vectors $\vec{n}_{a}, \vec{n}_{x}$ and $\vec{n}_{a}^{*}$ form a third plane according to the law of reflection. More specifically, the 
angle between $\vec{n}_{a}$ and $\vec{n}_{x}$, the incidence beam angle, and the angle between $\vec{n}_{x}$ and $\vec{n}_{a}^{*}$, the reflected beam angle, are equal according to this law. For example, this angle for the x-mirror when at its initial rest position is $45^{\circ}$. As a result, for convenience, $\vec{n}_{a}^{*}$ can be expressed in terms of $\vec{n}_{x}$ and $\vec{n}_{\alpha}$. Firstly, $\vec{n}_{a}$ is decomposed into components, $\vec{n}_{a_{1}}$ which is parallel to $\vec{n}_{x}$, and $\vec{n}_{a_{2}}$ which is perpendicular to $\vec{n}_{x}$ :

$$
\begin{aligned}
& \vec{n}_{a}=\vec{n}_{a_{1}}+\vec{n}_{a_{2}} \\
& \vec{n}_{a_{2}}=\vec{n}_{a}-\vec{n}_{a_{1}}
\end{aligned}
$$

The reflection caused by the x-mirror inverts the direction of $\vec{n}_{a_{1}}$ and retains the direction of $\vec{n}_{a_{2}}$. Decomposing $\vec{n}_{a}^{*}$ into components using the terms expressed in Equation 5 results in the following:

$$
\vec{n}_{a}=-\vec{n}_{a_{1}}+\vec{n}_{a_{2}}
$$

$\vec{n}_{a_{1}}$ can be rewritten according to the following equation:

$$
\vec{n}_{a_{1}}=\left(\vec{n}_{a} \cdot \vec{n}_{x}\right) \cdot \vec{n}_{x}
$$

Substituting Equation 8 into Equation 6, and subsequently Equation 8 and the modified Equation 6 into Equation 7, results in $\vec{n}_{a}^{*}$ being expressed in terms of $\vec{n}_{x}$ and $\vec{n}_{a}$ :

$$
\vec{n}_{\alpha}^{*}=-2\left(\vec{n}_{a} \cdot \vec{n}_{x}\right) * \vec{n}_{x}+\vec{n}_{a}
$$

The scalar, $\delta_{2}$, is given by:

$$
\delta_{2}=\frac{\vec{n}_{y} \cdot\left(\vec{R}_{o_{z}}-\vec{R}_{\hat{i}_{1}}\right)+D}{\vec{n}_{y} \cdot \vec{n}_{a}^{x}}
$$

such that $\vec{n}_{y}$ is the normal vector of the y-mirror's surface and $\vec{R}_{o_{z}}$ is the vector from the origin of the GCS to a point along rotation axis of the y-mirror. In order to calculate $\vec{n}_{y}$, the same procedure is used as in Equation 3. Equation 4 to Equation 10 are repeated for the y-mirror and when striking the f-theta lens aperture. To simulate the rotation of the mirrors, it is necessary to use a rotation matrix. As the mirror rotates, $\vec{P}_{x_{1}}, \vec{P}_{x_{2}}, \vec{P}_{y_{1}}$, and $\vec{P}_{y_{2}}$ are reconfigured by this rotation matrix such that $\vec{n}_{x}$ and $\vec{n}_{y}$ are subsequently recalculated. In effect, the reflected beam which is dependent on $\vec{n}_{x}$ and $\vec{n}_{y}$ can be manipulated based on the angle of rotation. This rotation matrix rotates about a user defined axis and point other than the axes or zero point of the GCS. This is because neither, the axis of rotation of each mirror lay coincident to the GCS axes, nor are they parallel as in the case of the x-mirror (rotated $11.5^{\circ}$ about the $\mathrm{x}$-axis). Firstly, the following unit vector defines the axis of rotation of either the $\mathrm{x}$-mirror or the y-mirror:

$$
\vec{u}=\left(\vec{u}_{x}, \vec{u}_{y}, \vec{u}_{z}\right) \text { where } u_{x}^{2}+u_{y}^{2}+u_{z}^{2}=1
$$

Secondly, the following $3 \times 3$ rotation matrix is used to rotate about an arbitrary axis in the $\mathrm{x}-, \mathrm{y}-$ and $\mathrm{z}-$ directions: 


$$
r=\left[\begin{array}{ccc}
\cos \alpha+u_{x}^{2}(1-\cos \alpha) & u_{x} u_{y}(1-\cos \alpha)-u_{z} \sin \alpha & u_{x} u_{z}(1-\cos \alpha)+u_{y} \sin \alpha \\
u_{y} u_{x}(1-\cos \alpha)+u_{z} \sin \alpha & \cos \alpha+u_{y}^{2}(1-\cos \alpha) & u_{y} u_{z}(1-\cos \alpha)-u_{x} \sin \alpha \\
u_{z} u_{x}(1-\cos \alpha)-u_{y} \sin \alpha & u_{z} u_{y}(1-\cos \alpha)+u_{x} \sin \alpha & \cos \alpha+u_{z}^{2}(1-\cos \alpha)
\end{array}\right]
$$

such that $\alpha$ is the angle of rotation of the mirror ranging from $-11.25^{\circ}$ up to $+11.25^{\circ}$. The mirrors rotate about the end point of $\vec{R}_{o}$ and $\vec{R}_{\sigma_{\mathrm{z}}}$ for the x- and y-mirror respectively. This results in the final $4 \times 4$ rotation matrix which now considers a defined rotation point other than the zero point of the GCS. For the $\mathrm{x}$-mirror as an example:

$$
R=\left[\begin{array}{cccc}
r_{00} & r_{01} & r_{02} & R_{o_{x}} r_{00} R_{o_{x}}-r_{01} R_{o_{y}}-r_{02} R_{o_{z}} \\
r_{10} & r_{11} & r_{12} & R_{o_{y}} r_{10} R_{o_{x}}-r_{11} R_{o_{y}}-r_{12} R_{o_{z}} \\
r_{20} & r_{21} & r_{22} & R_{o_{z}} r_{20} R_{o_{x}}-r_{21} R_{o_{y}}-r_{22} R_{o_{z}} \\
0 & 0 & 0 & 1
\end{array}\right]
$$

For example, the adjusted vector of $\vec{P}_{x_{1}}$ is carried out as follows given a $2^{\circ}$ angle of rotation:

$$
A=R(\alpha=0.035)\left[\begin{array}{c}
\overrightarrow{P_{x_{1}}^{T}} \\
1
\end{array}\right]
$$

such that $\vec{P}_{x_{1}}={ }_{3} A_{1}^{T}$. Now, calculations take a different approach and additional variables are outlined in Figure 3. For reference purposes, all calculations will be based upon the propagation of the laser beam from $(x 1, y 1)$ to a point on the marking field $(x 2, y 2)$ with respect to a local coordinate system (LCS).
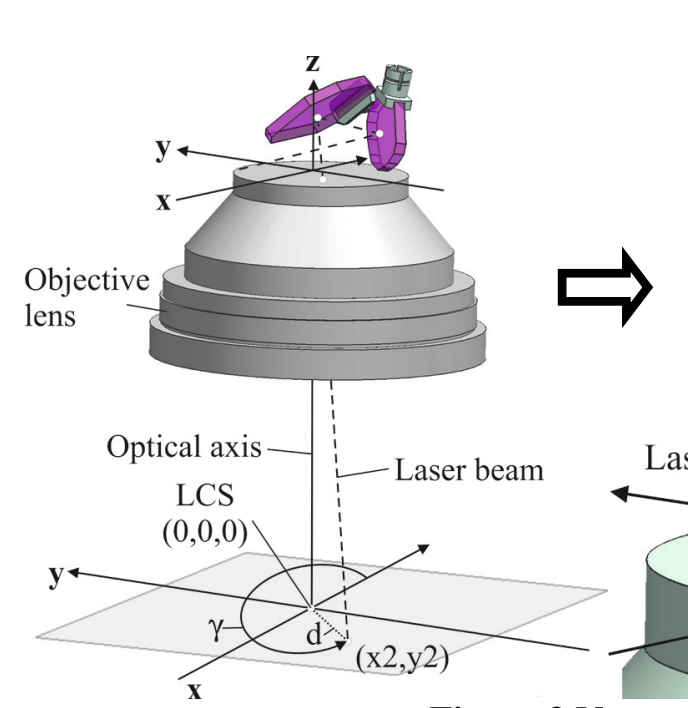

Figure 3 Vector and coordinate system notations

Initially, a vector is generated using the difference between $\vec{R}_{\tilde{i}_{\mathrm{z}}}$ and $\vec{R}_{\tilde{i}_{\mathrm{g}}}$ :

$$
\vec{v}_{\text {laser }}=\vec{R}_{i_{2}}-\vec{R}_{i_{g}}
$$

Subsequently, an arbitrary vector is created based on the end point of $\vec{R}_{\bar{i}_{\mathrm{I}}}$ denoted as coordinate position $(x 1, y 1)$. Its direction is parallel to the z-axis (i.e. optical axis) and normal to the aperture of the f-theta lens. This vector is denoted as $\vec{u}_{\text {axis }}$. Accordingly, one can then determine the zenith angle measured from the vertical $\vec{u}_{\text {axis }}$ to $\vec{v}_{\text {laser }}$. This indicates the deflection angle from the optical axis: 


$$
\theta=\cos ^{-1}\left(\frac{\vec{u}_{\text {axis }} \cdot \vec{v}_{\text {laser }}}{\left\|u_{\text {axis }}\right\|\left\|v_{\text {laser }}\right\|}\right)
$$

The azimuthal angle, $\gamma$, measured counter-clockwise from the $\mathrm{x}$-axis is dependent at which quadrant the point $(x 1, y 1)$ strikes the $\mathrm{f}$-theta lens aperture. Therefore, given the fact that the azimuthal angle must be calculated in the range of 0 to $2 \pi$ so that a full image can be achieved, the following piecewise function expresses this concept:

$$
\gamma=\left\{\begin{array}{lll}
\tan ^{-1}\left(\frac{y 1}{x 1}\right) & x>0 \text { and } y>0 & \text { Quadrant } I \\
\tan ^{-1}\left(\frac{y 1}{x 1}\right)+\pi & x<0 \text { and } y>0 & \text { Quadrant } I I \\
\tan ^{-1}\left(\frac{y 1}{x 1}\right)-\pi & x<0 \text { and } y<0 & \text { Quadrant } I I I \\
\tan ^{-1}\left(\frac{y 1}{x 1}\right)+2 \pi & x>0 \text { and } y<0 & \text { Quadrant } I V
\end{array}\right.
$$

The angle $\gamma$ with respect to point $(x 1, y 1)$ is equal for point $(x 2, y 2)$. To take into account the effect of the f-theta objective lens in terms of the laser beams position as it strikes the marking field, the ftheta condition is used. This implies that the use of an f-theta objective lens results in the displacement of the focal spot from the optical axis, $d$, to be linearly proportional (proportionality factor is the lens focal length, $f_{\text {lens }}$ ) to the deflection angle, $\theta$, i.e. $d=f_{\text {lens }} \theta$. This equation is expressed in terms of polar coordinates and can be converted to Cartesian coordinates as follows:

$$
\begin{aligned}
& x 2=f_{\text {lens }} \theta \cos (\gamma) \\
& y 2=f_{\text {lens }} \theta \sin (\gamma)
\end{aligned}
$$

\section{$3 \quad$ Results and Discussion}

The principles explained in Section 2 give the position of one point on the marking field given a defined angle of rotation of each respective mirror. Hence, it only takes into account a laser beam striking two stationary mirrors. In fact, in a laser scanhead, these mirrors move about its axis back and forth, $\alpha= \pm 11.25^{\circ}$, in order to generate the desired image (e.g. barcode, pore structures). The subsequent section will depict the propagation of the laser beam on the lens aperture and marking field during the entire range of rotation of the mirrors.

\subsection{Marking field simulation}

Each point in the subsequent figures (Figure 4) represents a unique rotation of each mirror in which both mirrors rotate about their entire oscillating range (from $-11.25^{\circ}$ to $+11.25^{\circ}$ ) in increments of $2^{\circ}$. As shown in Figure 4a, one can observe the distortion caused by the mirrors thickness and beam displacement at the entrance surface of the f-theta lens. This is referred to as axis alignment error. Subsequently, in Figure $4 \mathrm{~b}$, one can observe the combination of pin cushion and barrel distortion as the beam propagates through the f-theta lens and strikes the marking field. This is referred to as lens image distortion. The magnitude of distortion varies according to the focal length of the objective lens. The offset points shown in Figure $4 \mathrm{c}$ are used to correct and shift the points in Figure $4 \mathrm{~b}$ to obtain the ideal marking field geometry shown in Figure 4d. This is referred to as passive correction in which the angles of the mirrors are adjusted by a calculated difference and thus the image is corrected. This means of correction, typically in the form of a correction file, is supplied by the manufacturer of the 
scanhead which depends mainly on the focal length of the f-theta lens and input diameter of the laser beam into the entrance aperture.
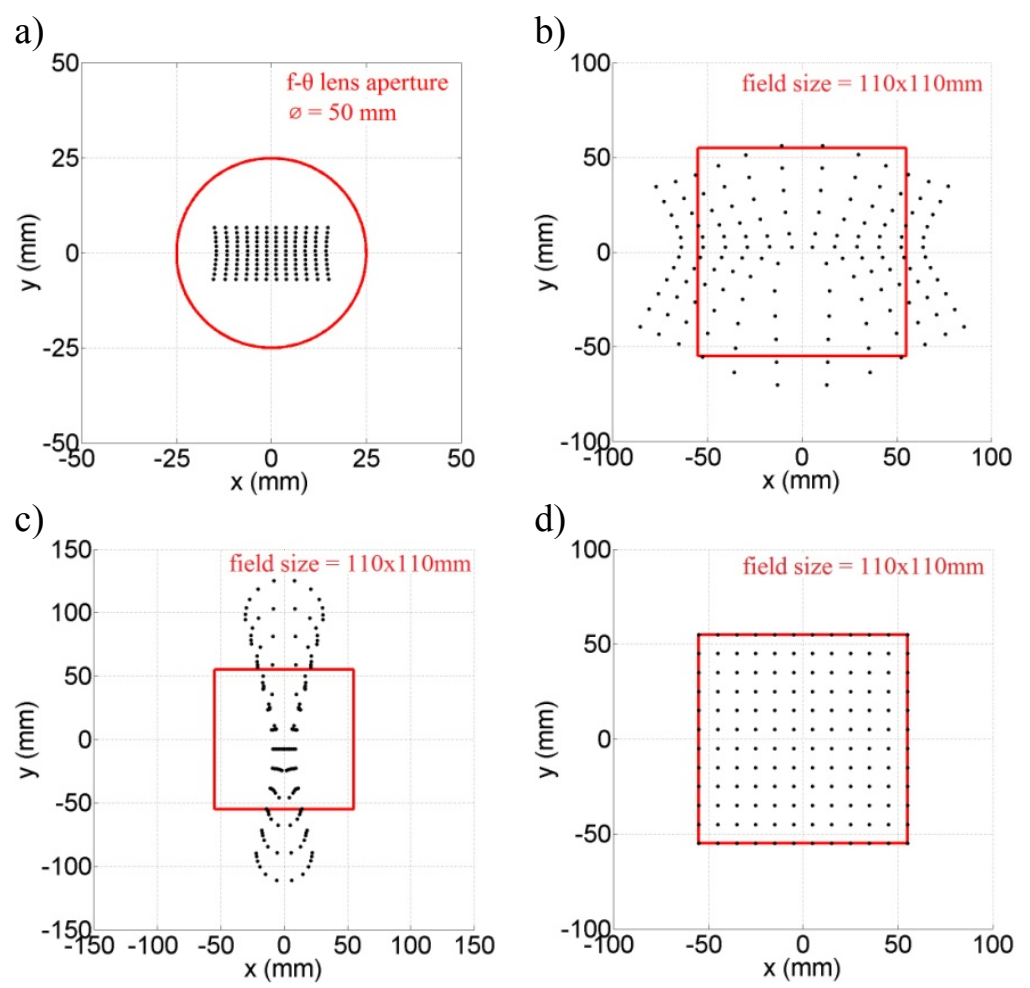

Figure 4 Points on the f-theta lens aperture (a), marking field after the f-theta lens (b), offset points (c), ideal marking field (d)

Now that both axis alignment error and lens image distortion have been discussed, it is interesting to understand what occurs during entrance aperture alignment errors. This is an extremely common error and its definition implies that the axis of the laser beam is misaligned with the axis of the scanhead's entrance aperture. This typically occurs when several bending mirrors are necessary to guide the laser beam into the aperture. In the presented case, the beam is shifted by $2 \mathrm{~mm}$ in the negative $\mathrm{z}$ - and $\mathrm{y}$ directions. This shift is denoted as $d i v_{z}$ and $d i v_{y}$. Furthermore, this shift occurs $300 \mathrm{~mm}$ away from the entrance aperture. With this information, one can calculate the angular deviation from the optical axis and substitute this angle in a rotation matrix to alter the unit vector of laser propagation, $\vec{n}_{\boldsymbol{\alpha}}$. Accordingly, one can observe this alignment error shown in Figure 5.

a)

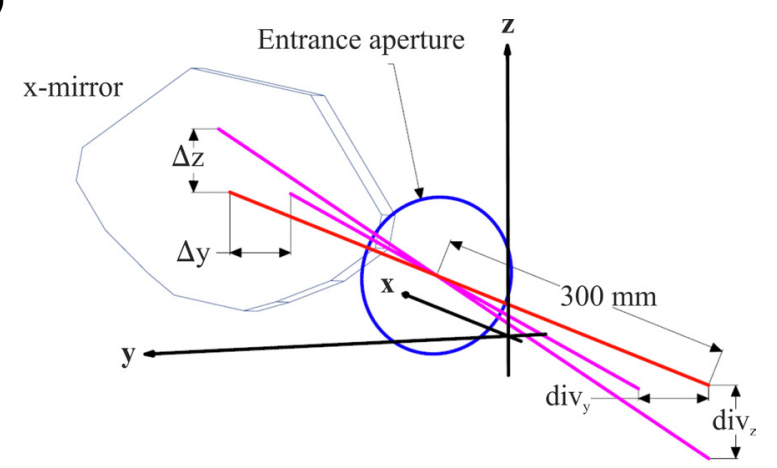

b)

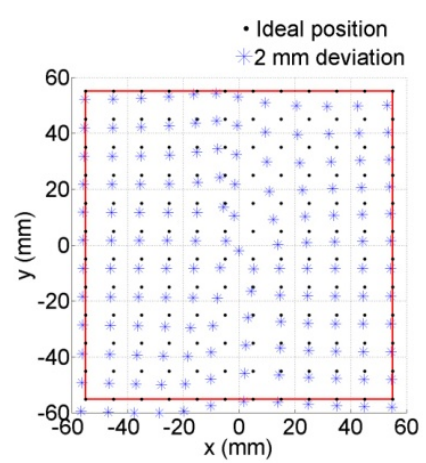

Figure 5 Schematic (a) and result (b) of a $2 \mathrm{~mm}$ shift in laser beam aperture alignment on the marking field

What can be deduced from this figure is that the points are shifted in the direction of the induced misalignment. Given that the deviation is directed to the bottom left hand corner, then this is replicated 
on the marking field with a shift in the bottom left hand corner. Sources and expressions of laser scanning errors are further explained by Luo et al. (2011) for the application of a range finder coupled with a scanning strategy. Although the errors are derived from a unique environment, the principles hold true and could be applied to the purpose of this publication. Axis alignment errors can be corrected by calculating a new set of offset points in-process or out-of-process by means of curve fitting, surface functions and other corrective transformations.

\subsection{Beam shape}

As explained in Section 2, the point of impact (i.e. the centre point of the beam) has been calculated, hence, Equation 20 - Equation 22 will characterize the shape of the beam in 2D and latter on the definition of ray tracing will characterize the beam in 3D. The following calculations are based on a Gaussian laser beam such that the calculated beam radius is based on when the intensity of the beam falls to $1 / e^{2}$ times the maximum value. Since all laser beams cannot achieve perfect collimation, the divergence half angle is calculated by:

$$
\theta_{\text {div }}=\frac{M^{2} \lambda}{\pi w}
$$

and the expansion in beam radius within the scanhead, given a propagation distance, denoted as $\delta$ (Equation 2 and Equation 10), is calculated by:

$$
w(\delta)=\delta \tan \left(\theta_{\text {div }}\right)+w
$$

such that $M^{2}, w$ and $\lambda$ is the beam quality factor, beam radius at the entrance aperture of the scanhead and wavelength of laser radiation respectively. The focal spot radius on the marking field given the beam radius at the aperture of the $\mathrm{f}$-theta lens aperture, $w_{\text {lens, }}$, is calculated by:

$$
w_{f}=\frac{M^{2} \lambda f_{\text {lens }}}{\pi w_{l e n s}}
$$

When the laser beam strikes the mirror, its shape will be elliptical since it strikes at an angle of incidence of $45^{\circ}$ for the $\mathrm{x}$-mirror and $38^{\circ}$ for the y-mirror with a oscillation range of $\pm 11.25^{\circ}$. One can use trigonometric principles to calculate the minor and major axes of the elliptical beam on each respective mirror and at each respective angle of incidence. The ellipticity of the beam is shown in Figure 6.

a)

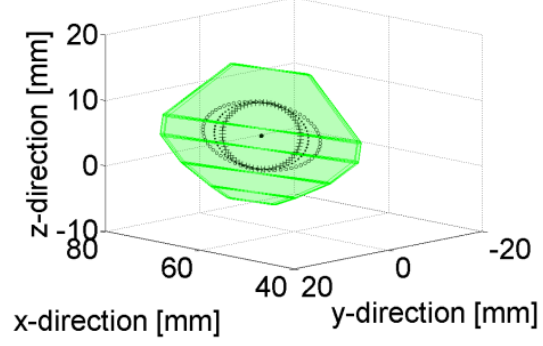

b)

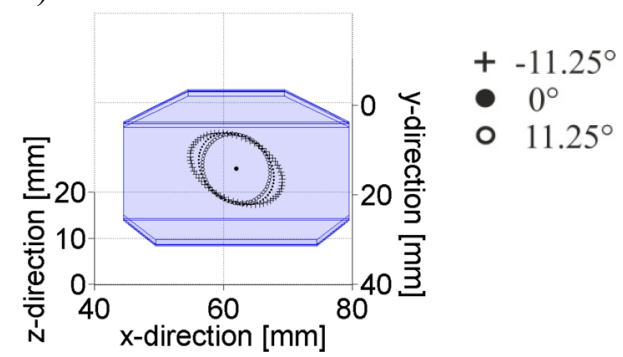

Figure 6 Simulation of beam shape normal to x-mirror (a) and y-mirror (b) throughout its oscillation range 


\subsection{Ray tracing}

It is known that light, more specifically a laser can be described by rays. Thus, the definition of ray tracing is the propagation of such rays through an optical system. This allows one to predict, design and optimize the given optical system. The path and geometry of a bundle of rays which comprises the laser beam is determined given that its propagation makes contact with a surface, e.g. a lens, beam splitter, prism, etc. Afterwards, laws of refraction, reflection, etc. are applied and the new propagation direction is retraced. Ray tracing provides an excellent means of visual understanding of laser beam propagation as it passes through several optical elements (Träger, 2007).

Since the beam radii, which were determined in Section 3.2, at each respective surface are known throughout its propagation path, i.e. entrance aperture, $x$-mirror surface, $y$-mirror surface, $f$-theta lens aperture and focal spot, the features of ray tracing can be simulated with the aid of Matlab ${ }^{\circledR}$ software as shown in Figure 7. The marking field dimensions are scaled for visual purposes. This is because the size and position of marking field is far greater in magnitude compared to the size of the mirrors.

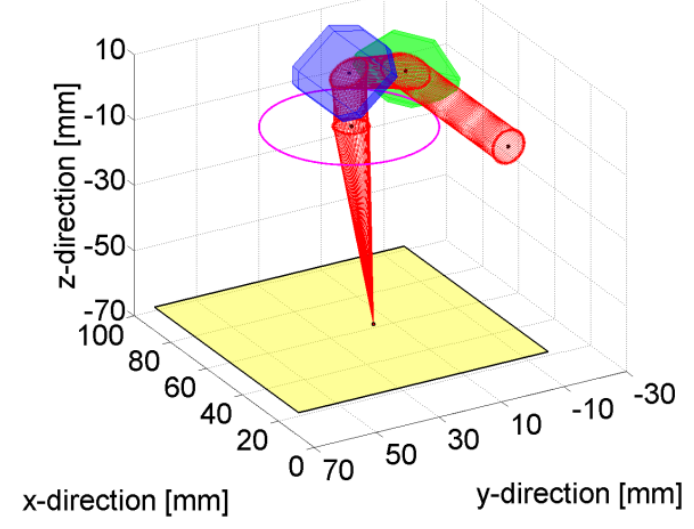

Figure 7 Simulation image of the visual functionality of a 2-axis laser scanhead

The propagation direction of each ray between the entrance aperture and $\mathrm{x}$-mirror surface, the $\mathrm{x}$-mirror surface and y-mirror surface, the y-mirror surface and f-theta lens aperture is based on $\vec{n}_{a}, \vec{n}_{a}^{z}$, and $\vec{n}_{a}^{v}$ respectively. The end and start position of each ray is determined from the shape and position of the beam radius at each respective surface. The shape of the beam based on Section 3.2, and the end and start position is based on the initial start position, $(5,5,5), i_{1}, i_{2}$, and $i_{3}$ or rather $(x 1, y 1)$ respectively.

\subsection{Gaussian Beam}

The purpose of this section is to further characterize the laser beam from simply being a bundle of rays to a more real characterization of electromagnetic radiation. Ray tracing proves valuable for beam propagation, but this section proves valuable to understand the evolved properties of the beam during propagation. This allows for future and more complex investigative topics such as heat interaction, optimized dielectric mirror design and damage thresholds. Since a scan system involves a laser beam in free space, three important assumptions will be made for which the following equations will be based on:

1. The beam's centre point does not lie at the origin, $\left(x_{0}, y_{0}\right) \neq(0,0)$

2. The beam's radius is not symmetric, i.e. elliptical, $w_{x}(\delta) \neq w_{y}(\delta)$

3. The minor or major axis of the beam is not parallel to the horizontal axis

The following Gaussian intensity function characterizes such a laser beam in three dimensions: 


$$
\begin{gathered}
f(x, y)=I * \exp \left(-\frac{\left(x-x_{0}\right)^{2}}{2 w_{x}^{2}(\delta)}-\frac{\left(y-y_{0}\right)^{2}}{2 w_{y}^{2}(\delta)}\right) \\
I=\frac{2 P}{\pi w_{x}^{2}(\delta)}+\frac{2 P}{\pi w_{y}^{2}(\delta)}
\end{gathered}
$$

such that, $I$, is the intensity amplitude at a defined propagation distance, which is conveniently expressed in terms of laser power, $P$, and the far field beam radius, $w(\delta)$, which is calculated from Equation 21. If one is interested in the near field beam radius (i.e. a focused beam), then one must refer to $w_{f}$, which is calculated from Equation 22. In this section, we are interested in the Gaussian intensity distribution on the mirror's surface, hence the beam is unfocused. Please note that when $\delta=0$, then the beam radius given no propagation distance is $w(0)=w$. Given a propagation distance, the radius typically increases since a laser beam can never achieve perfect collimation as previously stated. Equation 23 is generalized using the binomial theorem:

$$
f(x, y)=I * \exp \left(-l\left(x-x_{0}\right)^{2}+2 m\left(x-x_{0}\right)\left(y-y_{0}\right)+n\left(y-y_{0}\right)^{2}\right)
$$

such that one can then define the angle, $\beta$, between the horizontal axis and either the minor or major axis of the elliptical beam, given in the coefficients from Equation 25:

$$
\begin{aligned}
& l=\frac{\cos ^{2} \beta}{2 w_{x}^{2}(\delta)}+\frac{\sin ^{2} \beta}{2 w_{y}^{2}(\delta)} \\
& m=\frac{\sin 2 \beta}{4 w_{x}^{2}(\delta)}+\frac{\sin 2 \beta}{4 w_{y}^{2}(\delta)} \\
& n=\frac{\sin ^{2} \beta}{2 w_{x}^{2}(\delta)}+\frac{\cos ^{2} \beta}{2 w_{y}^{2}(\delta)}
\end{aligned}
$$

Given that $f(x, y)$ is the intensity distribution, then it could be normalized by dividing $f(x, y)$ with $I$. The above equations output the spatial Gaussian intensity distribution. It is more interesting though to observe the radial Gaussian intensity distribution as shown in Figure 8. The purpose is to compare the beam shape on the x-mirror shown in Fig. $6 \mathrm{a}$ with the Gaussian intensity distribution shown in Fig. 8 throughout its oscillating range. For simplicity purposes, the centre of the beam is located at the origin and $P=5 \mathrm{~W}$.

a)

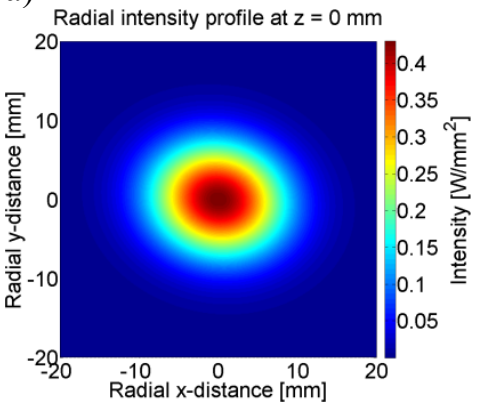

b)

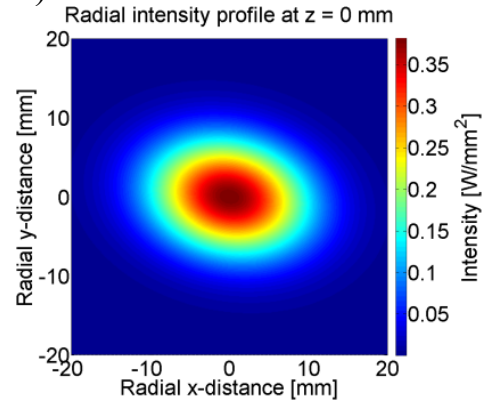

c)

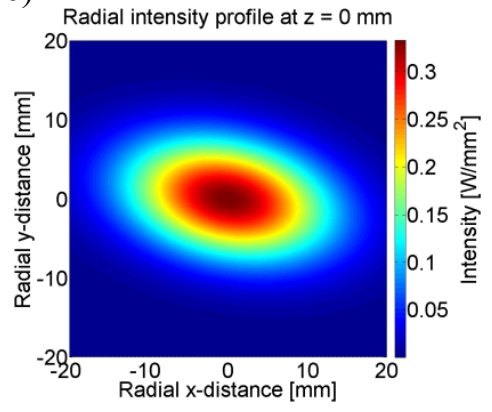

Figure 8 Radial intensity profiles when the laser strikes the x-mirror when $\alpha=-11.25^{\circ}$ (a), $0^{\circ}$ (b) and $+11.25^{\circ}(\mathrm{c})$ 
It is important to understand the characteristics of the laser beam intensity, firstly, to optimize the dielectric coating layers of the scanning mirrors so as to maximize reflection, and secondly, to be able to predict the thermal stresses which can deform the surface of the mirror. Shen et al. (2007) investigate this dilemma with respect to the laser forming of steel plates. The temperature gradient on a steel surface is derived from a Gaussian heat source and the deformation of the plate can therefore be predicted.

\section{Conclusions}

A two-axis laser scanhead was modelled using in-depth vector analysis then simulated using Matlab ${ }^{\circledR}$ software. Simulation results of the Raylase SuperScan-LD include: 1) understanding the generated images with respect to mirror oscillation, 2) results of errors caused by limitations of the scanhead and the user, 3) change in beam shape during mirror oscillation which is then related with the Gaussian beam intensity distribution, 4) and realizing beam propagation by means of ray tracing. The outlined principles can be further extended to model and simulate a three-axis laser scanhead by simply including information about a focus shifting lens. Furthermore, it is known that a change in mirror characteristics arises from high accelerations of the galvanometers and thermal influences from the laser beam and scanhead electronics. Thus, by changing the surface topography and geometry of the mirrors, one can more accurately understand the deviation of the focal spot on the marking field.

\section{Acknowledgements}

We would like to thank Raylase AG for hardware support and know-how to execute the simulation studies.

\section{References}

Bai, Y. and Wang D. (2011) 'Dynamic modelling of the laser tracking gimbal used in a laser tracking system,' Int. J. Modelling, Identification and Control, Vol. 12, Nos. 1/2, pp. 149-159.

Bessmeltsev V., Goloshevsky N. and Smirnov K. (2010) 'Specific features of controlling laser systems for micromachining of moving carriers,' Optoelectronics, Instrumentation and Data Processing, Vol. 46, No. 1, pp. 79-86.

Gandhi P. and Deshmukh S. (2010) 'A 2D optomechanical focused laser spot scanner: analysis and experimental results for microstereolithography,' Journal of Micromechanics and Microengineering, Vol. 20, pp. 1-11.

Garrido J. (2009) 'Models and Simulation' in Object Oriented Simulation: A Modeling and Programming Perspective, Springer Science + Business Media, New York, pp. 3-17.

Hafez M., Sidler T. and Salathé R. (2003) 'Study of the beam path distortion profiles generated by a two-axis tilt singlemirror laser scanner,' Optical Engineering, Vol. 42, No. 4, pp. 1048-1057.

Li Y. (2008) 'Beam deflection and scanning by two-mirror and two-axis systems of different architectures: a unified approach,' Applied Optics, Vol. 47, No. 32, pp. 5976-5985.

Li Y. and Katz J. (1995) 'Laser beam scanning by rotary mirrors. I. Modeling mirror-scanning devices,' Applied Optics, Vol. 34, No. 28, pp. 6403-6416.

Luo, Z., Liu H. And Wang F. (2011) 'Error analysis of robot detecting system with laser sensors in unknown environment,' Int. J. Modelling, Identification and Control, Vol. 12, Nos. 1/2, pp. 160-165.

Raylase AG. (2012) 2-Achsen Subsysteme zur Ablenkung von Laserstrahlen. http://www.raylase.com/de/downloads.php (Accessed 23 July 2012).

Shen H., Yao Z., Shi Y. And Hu J. (2007) 'The simulation of temperature field in the laser forming of steel plates,' Int. J. Modelling, Identification and Control, Vol. 2, No. 3, pp. 241-249.

Tamura S., Kim E., Close R. and Sato Y. (1994) 'Error correction in laser scanner three-dimensional measurement by twoaxis model and coarse-fine parameter search,' Pattern Recognition, Vol. 27, No. 3, pp. 331-338. 
Träger F. (Ed.), (2007) Springer Handbook of Lasers and Optics, Springer, New York.

Verboven, P. (1988) 'Distortion correction formulas for pre-objective dual galvanometer laser scanning,' Applied Optics, Vol. 27, No. 20, pp. 4172-4173. 